\title{
La teoría del valor trabajo y los precios en China
}

\author{
CÉSAR SÁNCHEZ \\ (UCA-El Salvador, UCM-Madrid) \\ cesarsan@servidor.unam.mx, cesarsanp@yahoo.com. \\ Everlan Elias Montibeler \\ (Universidade Federal de Mato Grosso Do Sul-Brasil) \\ everlam.montibeler@ufms.br
}

\section{Resumen}

El presente trabajo muestra la fuerte relación entre valores y precios en China con los datos de 2002. Además de mostrar esta relación con medidas de asociación como el coeficiente de correlación y determinación, se complementa el estudio con medidas de proximidad como la desviación absoluta media simple y ponderada, etc. Además se realiza un análisis simple de regresión entre los diferentes tipos de precios y se efectúa una estimación de las variables fundamentales para la economía oriental: tasa de beneficio, tasa de plusvalía, composición de capital simple y verticalmente integrada. Se concluye, en línea a otros trabajos que, el trabajo como requerimientos de trabajo directo e indirecto, explica prácticamente el $97 \%$ del movimiento de los precios de mercado, además, la tasa de beneficio de China parece mostrar en 2002 un nivel comparativamente mayor a países como España. China ha crecido su $P I B$ en términos reales sobre el $8 \%$, esta buena salud parece tener una sincronía con los niveles de su rentabilidad en esos años.

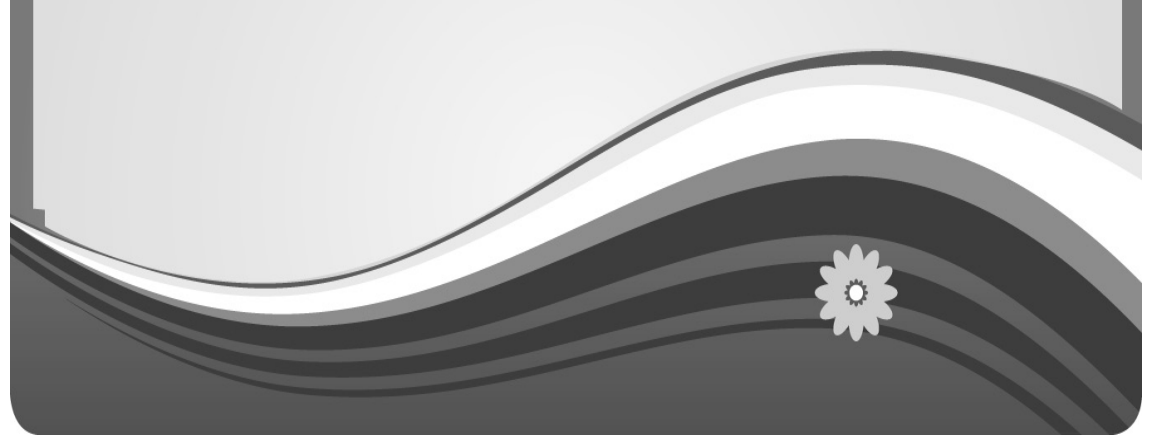




\section{Antecedentes}

E n los clásicos existe la idea de que los precios de las mercancías están determinados por las cantidades de trabajo (Meek, 1980). Esta idea va teniendo un desarrollo gradual. Desde Smith se tienen las nociones del trabajo comandado y, por otro lado, la de los esfuerzos que implica producir una mercancía. Ricardo es más preciso y desarrolla la idea de que el valor de una mercancía está determinado por el trabajo directo e indirecto incorporado en ella. Marx no sólo desarrolla la idea de Ricardo, sino que comprende el trabajo total (directo e indirecto) como el trabajo social y no sólo como trabajo del productor directo. Además Marx integra a su teoría del valor trabajo (TVT, en adelante), su teoría de la plusvalía, una comprensión totalmente ausente en Ricardo (Carcanholo, 2002 ).

Desde los ochenta, la idea del cálculo empírico de los valores ha resurgido desde la propuesta de Shaikh (1984). El autor utiliza para los Estados Unidos el esquema insumo-producto y los datos de Leontief para estimar los valores como los requerimientos directos e indirectos de trabajo. Estos requerimientos totales, estandarizados y expresados en dinero les denominó precios directos, también calculó los precios de producción sraffianos e hizo análisis de regresión y de medidas de distancia entre los diferentes precios, encontrando en general que, los valores explicaban y se aproximaban bastante bien a los precios corrientes (de mercado). Ochoa $(1984,1989)$ para el mismo país y basándose en Shaikh, calcula los valores, precios directos, de producción marxistas y de producción sraffianos utilizando las tablas insumo-producto (TIO, en adelante) para varios años, incorporando medidas de capital fijo en las estimaciones. Chilcote (1997) actualiza las tablas insumo producto para años más recientes y para países de la OCDE, además de que examina los Ilamados "valores alternativos", es decir insumos diferentes del trabajo que según algunos autores podrían ser igualmente explicativos como el trabajo. Chilcote profundiza como en Ochoa en formas diversas de cálculo de los precios de producción añadiendo gradualmente diferentes aspectos: capital fijo, rotación de capital, capacidad utilizada, de esta forma los precios de producción van acercándose conceptualmente más a los precios de mercado. Ambos autores utilizan diferentes medidas de distancia, y concluyen que los precios directos se aproximan bastante a los precios de producción e incluso aún más a los precios de mercado. Cockshott y Cotrell $(1995,1997)$ estiman con información de TIO del Reino Unido, obteniendo los diferentes tipos de precios y confirmando que los valores base como la electricidad, petróleo, química y la agricultura 
no explican más a los precios corrientes que los estimados por el trabajo (es decir, los determinados por la TVT). Guerrero (2000) en la misma línea que Chilcote, aplica la metodología para España (TIO 1986-1991), encontrando que los precios directos se acercan más a los de producción en la medida en que incorporamos al cálculo, capital fijo, rotación, etc. Guerrero realiza además un análisis teórico minucioso de las categorías desarrolladas y calculadas en este tipo de trabajos y confirma que las composiciones en valor del capital verticalmente integradas explican casi completamente las desviaciones entre precios directos y de producción, una idea teorizada por Marx en el tomo III de El capital. Para España, con las TIO 2000 y utilizando las estimaciones de vida media de los diferentes componentes del capital fijo publicadas por el Instituto Valenciano de Investigaciones Económicas, Sánchez y Nieto (2010) encuentran una fuerte asociación entre los valores y los diferentes tipos de precios (la desviación entre valores trabajo y precios de mercado es del $11 \%$ ) situando la tasa de beneficio española alrededor del $16 \%$. Por su parte Tsoulfidis y Manitis, (2002) han aplicado esta metodología para Grecia con información de TIO de 1970. Tsoulfidis junto con otros autores ha extendido este tipo de estudio a Corea, Japón, Canadá y China. Para el caso de China, la diferencia central de nuestro es que incorporamos datos de Stock de Capital Fijo, mientras Mariolis y Tsoulfidis (2009) utilizaron sólo capital circulante.

Después de los antecedentes de aplicación de la TVT a la relación entre valores y precios, por cuestiones de espacio, el presente sólo desarrollará los puntos concernientes a las fuentes estadísticas que se usaron en el estudio (2), a la relación entre valores y precios y el estudio econométrico entre los diferentes tipos de precios (3) y la estimación de las variables fundamentales en la economía China (4) por último se realizan unas breves conclusiones (5). La discusión sobre los aspectos econométricos, como el efecto del tamaño del sector, el sesgo posible el los estimadores del modelo planteado por Shaikh y otros aspectos relacionados con los precios, se dejan en una versión más extensa del mismo' ${ }^{1}$.

\section{Fuentes y los límites en las estadísticas en China}

Las TIO para China están disponibles para los años que van de 1987 hasta 2005. Estas tablas no se publican para todos los años, aunque ciertamente ha ido aumen- tado el grado de desagregación en que se presentan. La elección de trabajar con la tabla de 2002 fue porque es un año estable en el crecimiento de China, porque los 
datos son particularmente analizados por otros autores como $\mathrm{Holz}$ (2006) y porque servía para comparar mejor los resultados con otros trabajos sobre desviaciones entre precios. La mayoría de la literatura que ha estimado los precios y productividad de la economía China han encontrado problemas para conseguir una fuente confiable de estimación de Stock de capital. Más allá de los problemas estadísticos sobre el Stock de capital, también se tuvo que enfrentar al problema de la información sobre la fuerza de trabajo empleada por cada sector productivo. Esto es así, ya que el departamento de estadística en
China publica una metodología poco clara y detallada, sobre cómo están distribuidos y remunerados los trabajadores del campo y de la ciudad. Una buena parte de esta investigación consistió en estimar las estadísticas sobre trabajo y el Stock de capital en China. Para los datos sobre Stock de capital y trabajo, se utilizaron los destacables estudios del econometrista Gregory C. Chow (1993, 2006) y Chow y Kui-Wai Li (2002) y de Carsten A. Holz (2006) quienes realizan estimaciones pioneras sobre montos del capital en China. Las TIO se obtuvieron del Instituto oficial de estadística.

\section{La gran proximidad entre los valores y precios en China, 2002}

Con frecuencia se confunde a los estudios empíricos como un mero cúmulo de estadísticas desprovistas de teoría. No obstante, en la práctica científica, no todo intento teórico es un estudio científico y lo mismo vale para los estudios empíricos sino están respaldados por un modelo teórico a verificar. La hipótesis central en este trabajo es la de contrastar la afirmación de que el movimiento de los valores está determinando al movimiento de los precios. La metodología para obtener a los diferentes precios implica el uso de categorías y con- ceptos de la TVT que, en algunos casos por su complejidad, se ven necesariamente simplificadas para poder estimarse (vrg., la reducción de trabajo complejo a simple). Este tipo de estudios intenta contrastar una hipótesis como la anterior, dentro de la amplia TVT y bajo un modelo muy específico como el de Shaikh. Este es el contexto concreto de la corroboración empírica del presente trabajo ${ }^{2}$.

La idea del sistema de precios en Marx esquemáticamente es muy simple:

Valores $\rightarrow$ precios directos $\rightarrow$ precios de producción $\rightarrow$ precios de mercado 
La cadena causal plantea que los valores regulan a los precios de producción y estos a los de mercado, pero ¿cómo se calculan los valores?, los valores se determinan como los requerimientos de trabajo directos más indirectos.

En el tomo I Marx plantea los valores como la suma del capital constante más variable y la plusvalía $(\mathrm{W}=\mathrm{C}+\mathrm{V}+\mathrm{P})$. El capital constante representa al trabajo indirecto, mientras que el trabajo directo está representando al capital variable más la plusvalía $W=T I+T D)$. Matricialmente, esto puede aparentar ser más complicado, pero la idea sigue siendo la misma, en cambio, lo que se gana con este análisis es que se observan las relaciones intersectoriales de forma más detallada, algo que esconden precisamente el manejo de los simples agregados. Los valores trabajo se calculan entonces:

$$
\mathrm{I}=a_{o}(I-A-D)^{-1}
$$

Donde $A$ es la matriz de coeficientes técnicos (de 39×39) y D la matriz de coeficientes de depreciación, I es la matriz identidad y $a_{0}$ es el vector fila de los requerimientos de trabajo. Estos requerimientos totales de trabajo o valores por unidad de producción bruta $(\lambda)$ se multiplican por la expresión dineraria del valor y luego por la produc- ción de cada sector, obteniéndose los precios directos sectoriales. Un método especial se sigue para obtener los precios de producción y los precios sraffianos, los cuales se detallan en Sánchez y Nieto (2010) ${ }^{3}$.

Entonces es posible definir como una variable a explicar a los precios de mercado mediante los precios directos (los valores trabajo) y lo mismo puede realizarse con los precios de producción ${ }^{4}$. La figura 1 muestra la dispersión de los diferentes precios expresados en logaritmos naturales, respecto de los precios directos (línea de $45^{\circ}$ ). Cada punto representa un sector de los 39 utilizados en la TIO de China. Es ligeramente más dispersa la nube de puntos de los precios de mercado que la de los precios de producción. Pero en general se encuentra un buen ajuste para los diferentes precios; en otras palabras, el tiempo de trabajo directo más el indirecto (expresado en dinero), es una buena variable para explicar a los precios de producción (marxistas y sraffianos) y a los precios de mercado. 


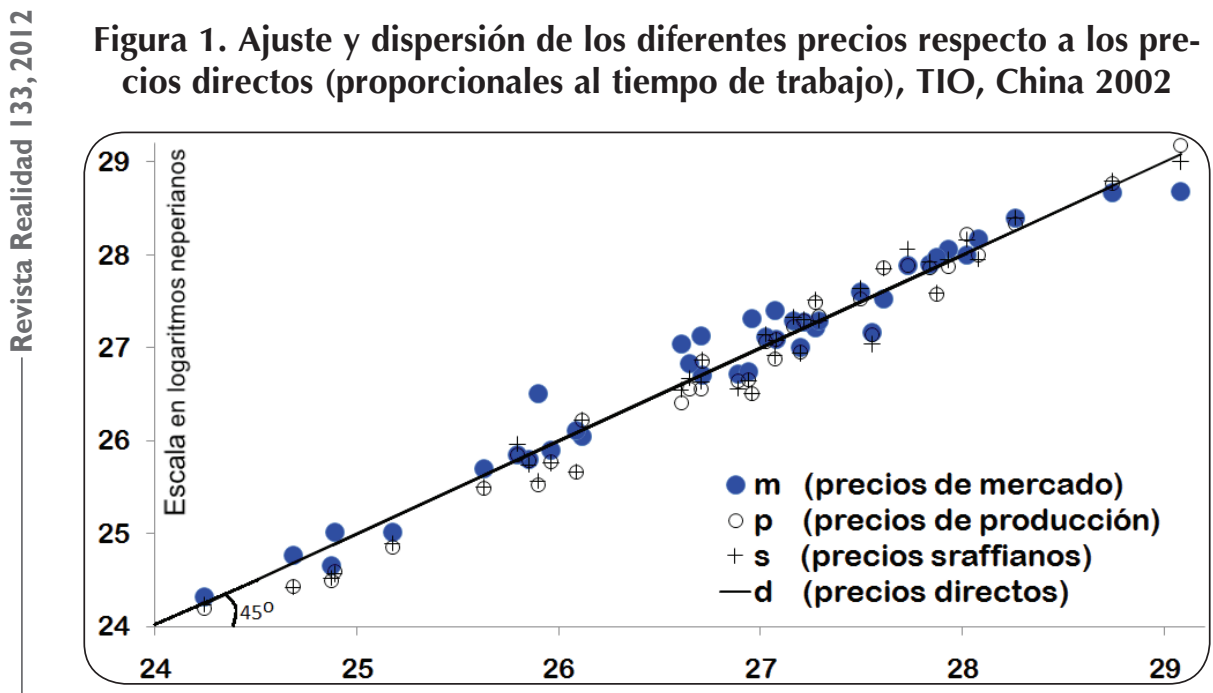

Si un sector tiene una producción de un múltiplo de 100 yuanes a precios directos, pero a precios de mercado 110 , entonces tomando como referencia los precios directos hay una desviación absoluta de $10 \%$; de este modo se calcula una "distancia" promedio para todos los sectores. La distancia absoluta media ponderada (DAMP) entre precios directos y de mercado $(\mathbf{d}, \mathbf{m})$ es de $14.19 \%$, mientras que la distancia de aquellos con los de producción $(\mathbf{d}, \mathbf{p})$ es de sólo $9.07 \%$, la proximidad entre precios de producción y sraffianos hacia los precios de mercado es incluso más lejana, $16.55 \%$ y $18.13 \%$ res- pectivamente, esto se cumple más o menos con los demás índices de distancia DAM (distancia absoluta media), DVN (distancia del vector normalizado) e incluso con los índices: "d", coeficiente de variación, CV y $\theta$ propuestos críticamente por Steedman \& Tomkins (1998); ellos sugieren utilizar estos últimos indicadores porque son independientes del numerario utilizado (ver tabla $1)$.

Como puede observarse, estas medidas no modifican las conclusiones anteriores, los precios directos y de producción se aproximan mucho a los precios de mercado ${ }^{5}$. 
Tabla 1. Medidas de desviación entre precios (\%)

\begin{tabular}{lrrrr}
\hline $\begin{array}{c}\text { Medidas de } \\
\text { desviación }\end{array}$ & \multicolumn{1}{c}{$(\mathbf{d}, \mathbf{m})$} & $\mathbf{( d , p )}$ & $(\mathbf{p}, \mathbf{m})$ & $(\mathbf{s}, \mathbf{m})$ \\
1. DAM & 14.19 & 12.01 & 16.54 & 18.50 \\
2. DAMP & 15.13 & 9.07 & 16.55 & 18.13 \\
3. DVN & 23 & 8.7 & 25.5 & 22.9 \\
4. Theil & 2.03 & 0.76 & 2.94 & 3.16 \\
5. Gini & 10.7 & 8.9 & 13. & 14.1 \\
6. C.V. & 19.25 & 15.53 & 23.25 & 24.58 \\
7. d & 18.99 & 15.39 & 22.79 & 24.04 \\
8. $\theta$ (en grados) & 10.89 & 8.82 & 13.08 & 13.81 \\
\hline
\end{tabular}

Nota: Como se ha señalado: $\mathbf{d}$ son los precios directos, $\mathbf{p}$ de producción, $\mathbf{s}$ de producción sraffianos y $\mathbf{m}$ los precios de mercado.

El análisis de regresión (tabla 2) entre precios muestra el siguiente orden de determinación: el crecimiento de los precios directos determina el movimiento de los de producción (98\%) y éstos determinan los de mercado (95\%). No obstante, los precios proporcionales al valor determinan el movimiento de los precios de mercado de forma muy significativa (96\%). Esto se corrobora estadística- mente mediante la mayor robustez de la $t$ calculada para la elasticidad de los modelos y para la explicación de los residuos mediante la prueba $F$ (obsérvese la mayor robustez de la relación $\mathbf{d}, \mathbf{p}$ y $\mathbf{d}, \mathbf{m}$, en ese orden). Los precios sraffianos explican satisfactoriamente a los de mercado, no obstante, lo hacen mejor los precios de producción y directos desde el enfoque marxista.

\section{Tabla 2. Regresiones simples log-log entre precios}

\begin{tabular}{|c|c|c|c|c|}
\hline Modelo & Estimación & $F$ & $\begin{array}{c}\text { C. Correlación } \\
\mathbf{R}\end{array}$ & $\begin{array}{l}\text { C. Determinación } \\
\text { R2 }\end{array}$ \\
\hline $\mathrm{mi}=\mathrm{f}(\mathrm{di})$ & $\begin{array}{l}\text { In mi }=0.64+0.97 \ln d i+u i \\
\text { t }(0.82) \\
(33.50)\end{array}$ & 1122.29 & $98.39 \%$ & $96.81 \%$ \\
\hline$p i=f(d i)$ & $\begin{array}{l}\text { In pi }=-0.59+1.04 \ln \mathrm{di}+\text { ui } \\
\mathrm{t}(-2.43)(50.37)\end{array}$ & 2537.38 & $99.27 \%$ & $98.56 \%$ \\
\hline$m i=f(p i)$ & $\begin{array}{l}\text { In mi }=0.99+0.91 \ln \mathrm{pi}+\mathrm{ui} \\
\mathrm{t}(2.54)(27.34)\end{array}$ & 747.49 & $97.61 \%$ & $95.28 \%$ \\
\hline $\mathrm{mi}=\mathrm{f}(\mathrm{si})$ & $\begin{array}{l}\ln \mathrm{mi}=1.24+0.89 \ln \mathrm{si}+\mathrm{ui} \\
\mathrm{t}(3.18)(26.74)\end{array}$ & 715.46 & $97.50 \%$ & $95.08 \%$ \\
\hline
\end{tabular}

Nota: Siendo $\mathrm{n}=39$ sectores y $\mathrm{k}=2$ el número de estimadores, el valor crítico $\mathrm{t}_{\alpha / 2}$ con grados de libertad $n-k=37 \approx 40$, entonces $t_{5 \% / 2}=2.02$. Como se sabe, en modelos como estos, el valor crítico de $\mathrm{F}$ al $5 \%$ de significancia es $\mathrm{F}_{(\mathrm{k}-1),(\mathrm{n}-\mathrm{k})} \approx \mathrm{F}_{1,40}=\mathrm{t}^{2}{ }_{5 \% / 2}=4.08$. De este modo, al ser mayores los valores de $\mathrm{t}$ y $\mathrm{F}$ calculados de sus valores críticos, son estadísticamente significativos: las variables explicativas y el modelo en general. 


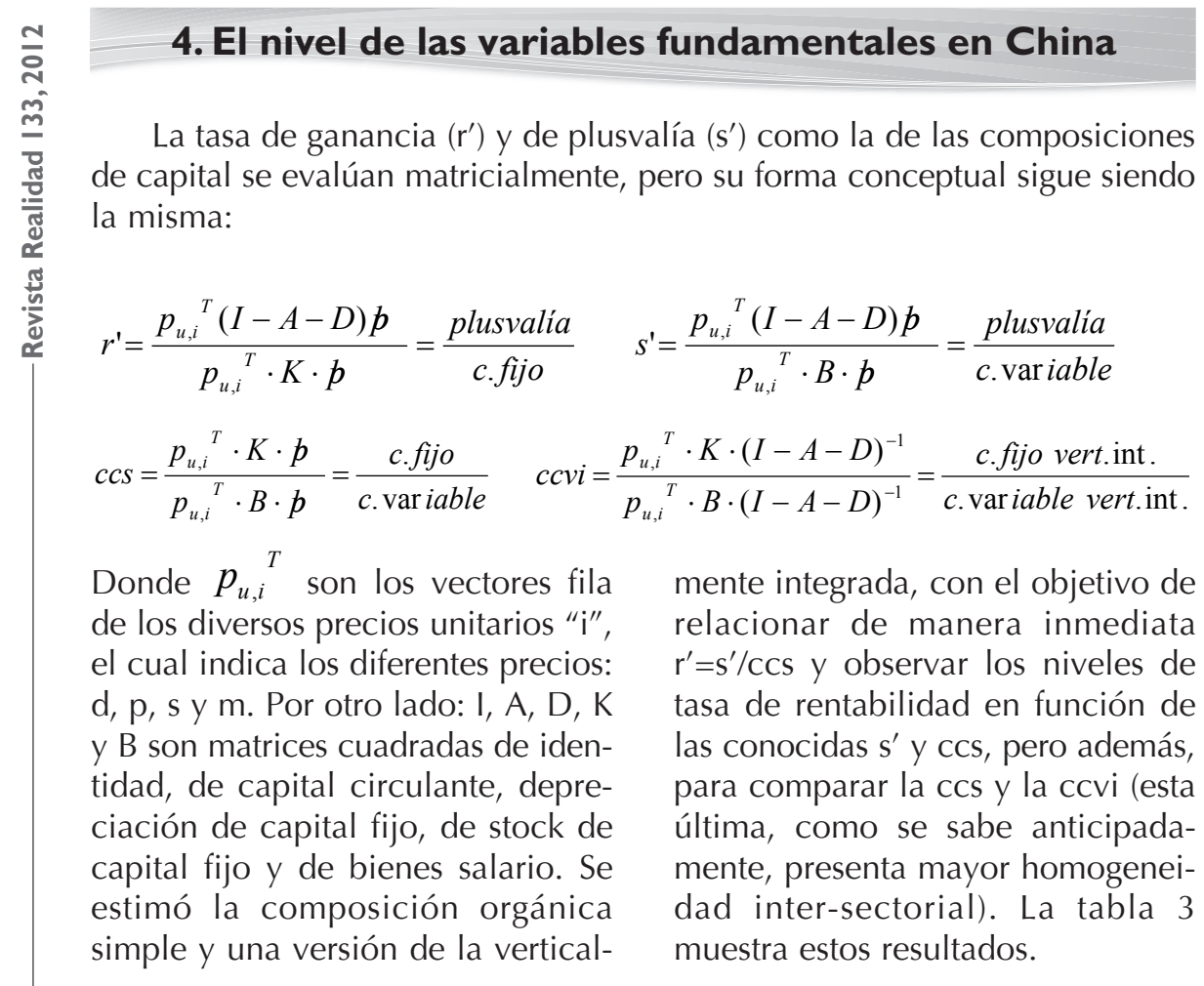

Tabla 3. Variables fundamentales marxistas en China 2002

\begin{tabular}{|c|c|c|c|c|c|c|}
\hline & $\begin{array}{l}\text { Precios de } \\
\text { mercado } \\
\text { (1) }\end{array}$ & $\begin{array}{l}\text { Precios } \\
\text { directos } \\
\text { (2) }\end{array}$ & $\begin{array}{l}\text { Precios } \\
\text { de pro- } \\
\text { ducción } \\
\text { (3) }\end{array}$ & $\begin{array}{l}\text { Precios } \\
\text { prod. } \\
\text { Sraffa } \\
\text { (4) }\end{array}$ & $(2) /(3)$ & $(2) /(1)$ \\
\hline Tasa de ganancia \% & 51.24 & 56.18 & 56.02 & 58.45 & 1.002 & 1.096 \\
\hline Tasa de plusvalía \% & 100.41 & 96.82 & 96.21 & 102.24 & 1.006 & 0.964 \\
\hline C. de capital simple & 1.9593 & 1.7231 & 1.7173 & 1.7492 & 1.005 & 0.882 \\
\hline $\begin{array}{l}\text { C.verticalmente } \\
\text { integrada }\end{array}$ & 2.2609 & 1.9884 & 1.9817 & 2.0185 & 1.003 & 0.876 \\
\hline
\end{tabular}

Las variables fundamentales en precios directos y de producción son casi idénticas, las diferencias son sólo un poco mayores entre precios de mercado y directos, tal como se concluía más arriba (co- lumna 5 y 6 de la tabla 3). La tasa de beneficio en valor parece ser más alta de lo que muestran los precios de mercado. Debe de recordarse que en el año 2002 la economía china estaba en franca expansión 
(creció en términos reales por encima del 8\%, Holz (2006: 113)). Es interesante apreciar que, con o sin ponderar el capital fijo, la tasa de ganancia china es mayor que la mostrada para otros países para más o menos el mismo año y con un cálculo con la misma metodología y medida en (r). En España, por ejemplo, con una $\mathrm{TIO}$ desagregada a 65 sectores en 2000 la rentabilidad es del: $16.09 \%, 17.29 \%$ y $13.38 \%{ }^{6}$ para precios de mercado, directos y de producción respectivamente (Sánchez y Nieto, 2010). Por otro lado, en Corea con una TIO desagregada a 27 sectores en 2000 y para el mismo orden de precios, las rentabilidades son: $11.6 \%, 13.6 \%$ y $13.3 \%$ (Tsoulfidis y Rieu, 2006). En cambio al comparar las tasas de plusvalía, mientras en China estas están entre el $96 \%$ y $100 \%$, en España se encuentran entre 66 y $76 \%$ y en Corea entre $73 \%$ y $86 \%$.

Con las salvedades y limitaciones que implica comparar las $\mathrm{TIO}$, en resumen se tiene que, China obtiene una mayor tasa de ganancia, con un menor nivel de composición de capital que otros países, pero que solventa, con un nivel mayor de explotación a sus trabajadores y que se refleja en una mayor tasa de plusvalía.

Esto es interesante, porque siguiendo la línea de Emmanuel (1972), Carchedi (1991) y Shaikh y Antonopoulos (1998), la ley del valor actúa a escala internacional y las altas tasas de ganancias son polos de atracción de capital.

\section{Conclusiones}

Los resultados de la gran cercanía entre precios en el caso de China se suma a los de otros trabajos recientes. La desviación absoluta media ponderada entre precios directos y de mercado es de $15.13 \%$, mientras que entre precios directos y de producción es de sólo $9.07 \%$. Estos resultados no se modifican al cambiar la medida de desviación o distancia, el sentido y el orden de las proximidades no se ve significativamente afectado. Parece ser que, para una de las economías más importantes del planeta, la fuerza de atracción que tienen los valores hacia los diferentes precios es bastante fuerte, concretamente las variaciones de los valores determinan las variaciones de los precios corrientes en un $97 \%$. El análisis de regresión entre los diferentes precios refuerza también esta conclusión, en la misma línea de lo que se ha encontrado en diversos países como EEUU, Grecia, Corea, España, etc.

Un último punto a remarcar es que, China parece mostrar una tasa de ganancia relativamente alta ya que, el rango de esta medida con los diferentes precios está entre $51 \%$ y $58 \%$ si se pondera sólo al capital fijo. Pero, incluso si se toma 
en cuenta al capital circulante, el rango baja entre $33 \%$ y $37 \%$, en definitiva, la rentabilidad en China está muy por encima de las rentabilidades encontradas con metodologías similares en países como Corea y España, que para un año similar sitúan sus rentabilidades por debajo del $18 \%$ para los diferentes precios. Nuevamente, parece que la TVT encuentra indicios sobre el mayor dinamismo en una economía como China.

\section{Bibliografía}

- Carcanholo, R. A., "Ricardo e o fracasso de uma teoria do valor". En: VII Encontro Nacional de Economia Política, 2002, Curitiba. Anais do VII Encontro Nacional de Economia Política. Curitiba, 2002.

- Carchedi, Guglielmo ., Frontiers of political economy. London: Verso. 1991, pp. 217-321.

- Chilcote, E., Interindustry structure, relative prices and productivity: an input-output study of the U.S. and O.E.C.D countries, tesis doctoral no publicada, New School for Social Research, New York, 1997.

- Cockshott, P. and Cottrell, A., "Labour time versus alternative value bases: a research note", Cambridge Journal of Economics, vol. 21, 1997, pp. 545-49.

- Cockshott, P. and Cottrell, A., "Does Marx need to transform?", in R. Bellafiore (Ed.) Marxian economics: A Reapparasal, vol. 2 Basingstoke, McMlllan st Martin's Press, 1998. Descargable.

- Cockshott, P., Cottrell, A. and Michaelson, G., "Testing Marx: some new results form UK data", Capital and Class, vol. 55, Spring, 1995, pp. 103-29.

- Chow, G.C. (1993): "Capital Formation and Economic Growth in China". Quarterly Journal of Economics 108. pp. 809-42. Descargable.

"New Capital Estimates for China: Comments". China Economic Review 17. 2006, pp. 186-92.

- Chow, G.C. and Kui-Wai Li (2002): "China's Economic Growth: 19522010", Economic Development and Cultural Change 51, 247-56. Descargable.

- Emmanuel, Arghiri., El intercambio desigual: ensayo sobre los antagonismos en las relaciones económicas internacionales. Ed. Siglo XXI, 1972, España. 
- Guerrero, D., Teoría del valor y análisis insumo-producto, manuscrito, 158 pp., 2000.

- Holz, C.A., "Measuring Chinese Productivity Growth, 1952-2005". 2006, disponible en SSRN: http://ssrn.com/abstract=928568

- Mariolis, T. y Tsoulfidis, L., "Decomposing the Changes in Production Prices into 'Capital-Intensity' and 'Price' Effects: Theory and Evidence from the Chinese Economy", Contributions to Political Economy, 2009.

- Marx, K., El Capital, Libros, I, II y III, Madrid, S. XXI, 2002.

- Meek, Ronald. Smith, Marx y después: Diez ensayos sobre el desarrollo del pensamiento económico, Ed. Siglo veintiuno, Madrid, 1980.

- Ochoa, E., "Labor Values and Prices of Production: An Interindustry Study of the U. S. Economy, 1947-1972", Ph. D. dissertation, Department of Economics, New School for Social Research, New York, 1984.

"Values, prices and wage-profit curves in the U.S. economy", Cambridge Journal of Economics, 13, 1989, pp. 413-429.

Shaikh, A., "The Transformation from Marx to Sraffa: prelude to a critique of the neo-ricardians", in E. Mandel and A. Freeman (eds.), Ricardo, Marx, Sraffa: The Langston memorial volume (London: Verso), 1984, pp. 43-84.

Valor, acumulación y crisis, Tercer Mundo Editores, Bogotá, 1990.

- Shaikh, A y Antonopoulos, R., "Explaining long-term exchange rate behavoir in the United States and Japan", Working Paper $n^{\circ} 250$. The Jerome Levy Economics Institute. 1998, pp. 1-28.

- Tsoulfidis, L. y Rieu, D., "Labor Values, Prices of Production and WageProfit Rate Frontiers of the Korean Economy", Seoul Journal of Economics, 2006.

- Tsoulfidis, L., Maniatis, T., "Values, prices of production and market prices: some more evidence form the Greek economy", Cambridge Journal of Economics, vol. 26, 2002, pp. 359-369.

- Steedman, I. and Tomkins, J., "On measuring the deviation of prices from values", Cambridge Journal of Economics, vol. 22, no. 3, 1998, pp. 379-85. 
una discusión más detallada sobre el anterior (vgr. sobre el sesgo en el modelo), se realiza en la versión más extensa de este trabajo.

5 Es relevante resaltar que en China se encuentra una proximidad mayor entre $(\mathbf{d}, \mathbf{p})$ respecto a $(\mathbf{d}, \mathbf{m})$, en relación a otros estudios como los de Ochoa (1989) para los Estados Unidos y los de Cockshott \& Cotrell (1998) para el Reino Unido. Esto es lo que, a priori, debería esperarse entre los precios directos y los de mercado pues aquí actúan otros elementos de competencia capitalista, como los movimientos en las rentas, movimientos generales de la demanda, impuestos, subsidios, etc.

6 Para China en cambio, ponderando de igual forma el capital fijo y circulante, la rentabilidad está entre el 33 y $37 \%$ para los diferentes precios. 